\title{
Motion parallax in depth and movement perception
}

\author{
FELIX E. GOODSON, STEVEN RITTER, and RANDY THORPE \\ DePauw University, Greencastle, Indiana 46135
}

\begin{abstract}
This experiment was designed to study the influence of motion parallax on depth and movement perception. Employing a model visual field with splotched backdrop, two planes of static gray balls and an intermediate moving red stimulus ball were presented while subjects were also under various conditions of lateral movement. Three hypotheses were advanced: (1) Viewed by a moving subject, a middle-ground object moving in synchrony with static objects in either a foreground or background plane will be judged as static and at the depth of the synchronous plane. (2) Viewed by a moving subject, a middle-ground object that is being shifted from synchrony with one plane to synchrony with the other plane will be judged as moving away from the original plane toward the other plane. (3) When moving out of synchrony with a given plane, a stimulus object will be seen as moving laterally (as well as shifting in depth) until it achieves synchrony with the other plane, at which time it will be perceived as static. The first two hypotheses were confirmed and qualitative data were obtained supporting the third.
\end{abstract}

This experiment was designed to study the influence of motion parallax on the perception of depth and motion. When a subject moves with respect to the environment, the rate at which the projection of an object moves across his retina varies inversely with the distance of the object (Braunstein, 1976). That is, nearer objects will be perceived as moving opposite to the direction of movement at. a progressively more rapid rate than will further objects. The reader can check this basic fact of motion parallax by moving his head back and forth while observing objects at various distances.

Evidence from previous research indicates that motion parallax is a cue to depth perception. Bourdon (1906) found that a subject whose head was fixated by a biting board could not resolve the relative depth of two luminous disks in a dark corridor, but such judgments were made without difficulty when the subject was permitted to move his head. In a more recent report (Gibson, 1959), subjects who viewed shadows of opaque disks moving at different velocities across an opaque screen saw them at different depths, and some were confused as to which was "nearer" and which was "farther."

The present study was designed to replicate such research in an experimental situation that approximated the conditions normally encountered by subjects while moving within the environment. To accomplish this, a moving stimulus ball located between a foreground and a background plane was presented to subjects who were moving laterally back and forth on a runway.

Reprint requests should be sent to the first author, Department of Psychology, DePauw University, Greencastle, Indiana 46135.

\section{Hypotheses}

(1) As viewed by a moving subject, a middle-ground object moving in synchrony with stationary objects in either a background or foreground plane will be perceived as not moving and at the depth of the plane with which it is moving in synchrony. (2) As viewed by a moving subject, a middle-ground object being shifted from synchrony with a foreground plane to synchrony with a background plane (or vice versa) will be seen as moving away from the depth of the original plane towards the depth of the other plane. (3) When moving out of synchrony with a given plane, the stimulus object will be seen as moving on its own until it achieves synchrony with the other plane, at which time it will be perceived as not moving.

\section{METHOD}

\section{Subjects}

Twenty undergraduate students from DePauw University (10 male and 10 female) volunteered to be subjects. All were screened for corrected or normal 20/20 vision.

\footnotetext{
Apparatus

Visual display. An open-faced rectangular black wooden frame measuring $12 \times 6 \times 3 \mathrm{ft}$ provided housing for the visual array. A total of 45 gray Styrofoam balls, ranging from $1 \frac{1}{2}$ to 3 in. in diameter, were strung on eight 10 -lb-test clear nylon lines in two planes perpendicular to the subject's line of sight. The planes were $30 \mathrm{in}$. apart, with the foreground set of 23 balls located at the front of the wooden frame and the background set of 22 balls located 6 in. in front of a black and white, randomly splotched, nonreflective surface.

A red Styrofoam ball, 2 in. in diameter, was used as a stimulus object, and was moved horizontally by a 1/15-hp reversible electric motor and pulley assembly, on a line equidistant from the two planes of Styrofoam balls. The speed of movement was regulated by a Variac connected to the motor. Subject transport. This apparatus included a $9 \times 2 \frac{1}{2} \mathrm{ft}$
} 
runway constructed of plywood. At one end a single pulley was mounted; at the other was a double pulley. An 18-ft V-belt, attached to a cart with a chair mounted on it, moved between the two pulleys. Guide supports of $1 / 2$-in. wood were fixed to the runway to stabilize lateral movement of the cart. The remaining pulley was connected by a 40-in. V-belt to a triple takedown speed-reduction unit. A 1/2-hp constant-torque variablespeed reversible electric motor was connected to this reduction assembly.

All apparatus were adjusted so that synchronization between the stimulus ball and the background and foreground planes was achieved when the stimulus ball moved at $1.15 \mathrm{~cm} / \mathrm{sec}$ while the chair moved at $24 \mathrm{~cm} / \mathrm{sec}$ at a distance of $4.55 \mathrm{~m}$ from the respective plane.

Lighting consisted of four $150-\mathrm{W}$ floodlights mounted in each of the four corners of the rectangular frame, behind white translucent plastic diffusing panels. A screen was used to obscure the visual field between trials. Two pairs of glasses, with one eye blacked out, allowed subjects to view the visual field monocularly with the preferred eye. An answer sheet containing five alternatives was used to record subjects' responses.

\section{Procedure}

Pretest treatment. Each subject was blindfolded, led into the room by the experimenter, seated in the mobile chair, and read the following instructions: "We are doing research in depth and motion perception. In a moment we will remove your blindfold and allow you to observe the experimental apparatus." The blindfold was removed from the subject's preferred eye and, while being moved back and forth once on the mobile chair, the subject was allowed to observe the two planes of Styrofoam balls without the presence of the stimulus ball. He was then asked whether the balls in the visual field separated themselves into a foreground and a background. If the answer had been no, the subject was to have been dropped from the experiment, but all subjects answered yes.

The subject was then told: "In the following trials you will see a red ball in the visual field. It is your job to note the behavior and location of the red ball in relation to the two planes you have just observed. You will be allowed to make 16 observations. Between each of these observation trials, you will be given this card on which you will check what you observed. Please examine this card carefully. Remember that you should check what you actually observed, not what you think the experimenter might want."

Each subject was then handed a card and allowed to examine it for $2 \mathrm{~min}$. The card instructed the subject to check only one of the five alternatives: (1) The red ball was in the foreground. (2) The red ball was in the background. (3) The red ball shifted from the foreground to the background. (4) The red ball shifted from the background to the foreground. (5) I don't know.

The final instructions were then read. "After you have made your response on the response card, you will hear me begin to count. I will count from one to five. On one you will close your eyes just as you feel yourself begin to move; on two open your eyes and view the visual field; on three and four continue to observe the visual field, and on five close your eyes."

This technique was chosen after prolonged investigation with pilot subjects, using different kinds of presentation procedures and apparatus. Although imperfect, it allowed approximately $4 \mathrm{sec}$ of exposure during each period of optimal synchronization or shift.

Test trials. The subject was then given 16 trials, with the four test conditions randomly presented and the two directions of movement alternated. The four presentation conditions were:
(1) the stimulus ball moving in synchrony with the background, (2) the stimulus ball moving in synchrony with the foreground, (3) the stimulus ball shifting from synchrony with the foreground to synchrony with the background, and (4) the stimulus ball shifting from synchrony with the background to synchrony with the foreground.

Each subject was given $30 \mathrm{sec}$ between trials to make his response on the response card and was allowed to rest for $10 \mathrm{~min}$ between the two blocks of eight trials. Between trials, the subject's view of the visual display apparatus was obscured by a screen. The screen was removed at the count of one and replaced at the count of five.

\section{RESULTS}

When the red stimulus ball was synchronized with either the foreground or background, the subjects reported on $86.88 \%$ of the trials that it was located at that particular plane. When the red ball was shifted from synchrony with one plane to that of the other, the subjects reported that it changed depth in the predicted direction on $70.63 \%$ of all trials. A nonparametric Wilcoxon matched-pairs signed-ranks test was applied to these percentages as against a $20 \%$ expected occurrence. In both cases the differences between observed and expected frequencies was significant at the .01 level of confidence.

These results indicate that motion parallax is an important cue for depth perception. When viewed by a moving observer, an object moving synchronously with a particular ground is seen as static and at the same depth as that ground. The data from the shift trials was perhaps even more impressive. On such trials subjects perceived the stimulus ball as actually moving away from or toward them as it changed synchrony from one plane to the other. The shift trials also provided data that suggest movement parallax is an important cue for movement perception. When the stimulus ball began to move out of synchrony with a given plane, subjects immediately saw the ball start to move laterally on its own. If the stimulus ball was shifting from background to foreground synchrony, most subjects reported that it speeded up in a forward direction. When the stimulus ball was shifting from foreground to background synchrony, many subjects saw it moving backward even though it was moving forward.

\section{REFERENCES}

Bourdon, B. La perception visuelle de l'espace. Paris: Schleicher, 1906.

Braunstein, M. L. Depth perception through motion. New York: Academic Press, 1976.

Gibson, E. J. Motion parallax as a determinant of perceived depth. Journal of Experimental Psychology, 1959, 58, 40-51.

(Received for publication July 24, 1978.) 